\title{
GMR
}

\section{Association between IL6 polymorphism and risk of cerebral infarction}

\author{
Y.H. Han \\ Corresponding author: Y.H. Han \\ E-mail: hanyuhua_xxch@126.com \\ Genet. Mol. Res. 14 (4): 16438-16443 (2015) \\ Received August 15, 2015 \\ Accepted October 2, 2015 \\ Published December 9, 2015 \\ DOI http://dx.doi.org/10.4238/2015.December.9.14
}

Department of Neurology II, Xinxiang Central Hospital, Xinxiang, Henan, China

\begin{abstract}
We conducted a case-control study to investigate the influence of IL6 -174G/C (rs1800795) and -572C/G (rs1800796) genetic variants on the development of cerebral thrombosis in a Chinese population. This study included 305 cerebral infarction patients and 326 control subjects enrolled between May 2012 and May 2014. The genotyping of IL6 -174G/C (rs1800795) and -572C/G (rs1800796) polymorphisms was performed using polymerase chain reaction combined with restriction fragment length polymorphism analysis. By using logistic regression, we found that when compared with the wildtype genotype, CC and GC+CC IL6 -174G/C (rs1800795) genotypes were associated with an increased risk of cerebral infarction. Odds ratios (and 95\% confidence intervals) were calculated to be 3.10 (1.57-6.41) and $1.63(1.14-2.33)$ for the CC and GC+CC genotypes, respectively. In conclusion, our study suggests that the $\mathrm{CC}$ genotype and $\mathrm{C}$ allele of the IL6 -174G/C (rs1800795) polymorphism are associated with an increased risk of cerebral infarction.
\end{abstract}

Key words: IL6; Polymorphism; Cerebral infarction 


\section{INTRODUCTION}

Stroke is a complex-trait disease and a leading cause of morbidity and mortality worldwide (Donnan et al., 2008). In addition, it has recently been reported that stroke-related mortality has become the leading cause of death in China (Liu et al., 2011). Cerebral infarction constitutes the most common type of stroke, making up an estimated 43.7 to $78.9 \%$ of strokes in the Chinese population (Liu et al., 2007). The etiology of cerebral infarction involves many factors such as hypertension, diabetes, hyperlipidemia, vasculitis, atherosclerosis, and genetic influences (Ionita et al., 2005; Dichgans, 2007; Meschia et al., 2011).

An increasing amount of evidence has shown that inflammation plays a critical role in the pathogenesis of atherosclerosis and cerebral infarction (Stoll and Bendszus, 2006), and that several cytokines are associated with the arterial wall inflammatory process. Variations in genes related to the inflammatory system may alter the pattern of proinflammatory cytokine production, and thus the development of cerebrovascular disease, affecting predisposition and prevalence (Andreotti et al., 2002).

Interleukin 6 (IL6) is a cytokine with many important functions and is involved in several contradictory processes. It plays key roles in a wide spectrum of target cells in the immune response, hematopoiesis, neural differentiation, and the acute phase reaction (Schoester et al., 1994). IL6 is reported to be involved in the inhibition of lipoprotein lipase activity and the stimulation of lipolysis, and thus may affect the pathogenesis of cerebral infarction (Shenhar-Tsarfaty et al., 2010; Saiki et al., 2013). Previous studies have investigated the association between IL6 polymorphisms and the development of cerebral infarction, but results have been inconsistent (Balding et al., 2004; Yamada et al., 2006; Shenhar-Tsarfaty et al., 2010; Qi et al., 2014). Therefore, we conducted a case-control study to investigate the effect of IL6 -174G/C (rs1800795) and -572C/G (rs1800796) genetic variants on the development of cerebral thrombosis in a Chinese population.

\section{MATERIAL AND METHODS}

\section{Study population}

This case-control study included 305 cerebral infarction patients and 326 control subjects enrolled between May 2012 and May 2014. All cerebral infarction patients were diagnosed by computed tomography or magnetic resonance imaging according to the World Health Organization cerebral infarction criteria. The diagnostic criteria were rapidly developing clinical signs of focal or global disturbance of cerebral function lasting more than $24 \mathrm{~h}$ with no apparent cause besides that of vascular origin. Patients with intracranial hemorrhage, transient ischemic attacks, peripheral vascular disease, thrombosis-related disease, brain tumors, or brain trauma were excluded from the study.

Cancer-free control subjects were randomly selected from individuals visiting the same hospital for health checkups. Control subjects with a history of strokes, peripheral vascular disease, or cancer were excluded from our study.

Clinical and demographic information regarding cerebral infarction patients and control subjects was collected from medical records. This data included sex; gender; body mass index (BMI); tobacco and alcohol consumption; levels of triglycerides, cholesterol, low-density lipoproteins (LDLs), and high-density lipoproteins (HDLs); and whether subjects had hypertension or diabetes. Written informed consent was obtained from each subject before being enrolled in the study group. This work was approved by the Institute Research Ethics Committee of Xinxiang Central Hospital. 


\section{Genotyping}

Each patient and control subject provided a $5 \mathrm{~mL}$ peripheral venous blood sample after enrollment. The collected blood samples were stored at $-20^{\circ} \mathrm{C}$ until use. Genomic DNA was extracted from peripheral blood using a TIANamp Blood DNA kit (Tiangen Biotech Co., Ltd., Beijing, China). The genotyping of IL6 -174G/C (rs1800795) and -572C/G (rs1800796) polymorphisms was performed using polymerase chain reaction (PCR) combined with restriction fragment length polymorphism analysis. The forward and reverse primers used to amplify IL6 -174G/C (rs1800795) were 5'-AGC CTC CGT GAC CAA ATA AG-3' and 5'-GGC GCT GAT TCC AAA GGT TA-3', respectively. Those for IL6 -572C/G (rs1800796) were 5'-GGA GAC GCC TTG AAG TAA CTG C-3' and 5'-GAG TTT CCT CTG ACT CCA TCG CAG-3', respectively. Amplification was performed using the following cycling program: an initial denaturation step at $95^{\circ} \mathrm{C}$ for $5 \mathrm{~min}$, then 30 cycles of denaturation at $95^{\circ} \mathrm{C}$ for $30 \mathrm{~s}$, annealing at $62^{\circ} \mathrm{C}$ for $45 \mathrm{~s}$, and extension at $72^{\circ} \mathrm{C}$ for $30 \mathrm{~s}$, followed by a final extension at $72^{\circ} \mathrm{C}$ for $5 \mathrm{~min}$. PCR products were visualized on a $2 \%$ agarose gel stained with ethidium bromide and exposed to ultraviolet light.

\section{Statistical analysis}

Statistically significant differences between cases and controls for demographic characteristics were assessed by the chi-square test. The association between IL6 -174G/C (rs1800795) and -572C/G (rs1800796) polymorphisms and risk of cerebral infarction was analyzed by calculating odds ratios (ORs), 95\% confidence intervals ( $95 \% \mathrm{Cls}$ ), and corresponding P-values. Gene-environment interactions were estimated by stratified analysis of demographic and clinical characteristics. Deviation from Hardy-Weinberg equilibrium for the polymorphisms under investigation was evaluated by comparing expected to observed genotype frequencies using the chi-square test. All P-values were two-sided, and those of less than 0.05 were considered to signify significant differences. All statistical analyses in this study were performed using SPSS software, version 16.0 (SPSS, Chicago, IL, USA) for Windows.

\section{RESULTS}

The demographic and clinical characteristics of cerebral infarction patients and control subjects are presented in Table 1. No significant difference was found between the two groups in terms of sex and age $(P>0.05)$.

When compared with control subjects, the cerebral infarction patients were more likely to have a higher BMI, suffer from diabetes mellitus and hypertension, have a habit of tobacco and alcohol consumption, and show higher levels of LDL, HDL, and triglycerides $(P<0.05)$.

IL6 -174G/C (rs1800795) and -572C/G (rs1800796) genotype distributions were found to be consistent with Hardy-Weinberg equilibrium in the control group (Table 2). In the control group, the minor allele frequencies of these two single nucleotide polymorphisms (SNPs) were similar to those given in the National Centre for Biotechnology Information database (http://www.ncbi.nlm.nih.gov/snp).

Using logistic regression analysis, we found that individuals with IL6 -174G/C (rs1800795) $\mathrm{CC}$ and $\mathrm{GC}+\mathrm{CC}$ genotypes had an increased risk of cerebral infarction when compared with those carrying the wild-type genotype. The ORs (and 95\%Cls) were 3.10 (1.57-6.41) and 1.63 (1.142.33) for the CC and GC+CC genotypes, respectively (Table 2). However, no significant difference in cerebral infarction risk was found between variants of IL6 -572C/G (rs1800796). 
Table 1. Demographic and clinical characteristics of case and control subjects.

\begin{tabular}{|c|c|c|c|c|c|c|}
\hline & $\mathrm{Cl}$ cases $\mathrm{N}=305$ & $\%$ & Controls $\mathrm{N}=326$ & $\%$ & Chi-square or $t$-test & $P$ value \\
\hline Mean age, years & $60.15 \pm 10.70$ & & $59.30 \pm 10.25$ & & 1.02 & 0.15 \\
\hline$<55$ & 151 & 49.35 & 175 & 53.68 & & \\
\hline$\geq 55$ & 154 & 50.33 & 151 & 46.32 & 1.1 & 0.3 \\
\hline \multicolumn{7}{|l|}{ Gender } \\
\hline Male & 182 & 59.48 & 185 & 56.75 & & \\
\hline Female & 123 & 40.20 & 141 & 43.25 & 0.55 & 0.46 \\
\hline BMI, kg/m² & $25.10 \pm 2.85$ & & $23.50 \pm 3.10$ & & 6.74 & $<0.001$ \\
\hline \multicolumn{7}{|l|}{ Hypertension } \\
\hline No & 163 & 53.44 & 223 & 68.40 & & \\
\hline Yes & 142 & 46.56 & 103 & 31.60 & 14.85 & $<0.001$ \\
\hline \multicolumn{7}{|l|}{ Diabetes mellitus } \\
\hline No & 218 & 71.48 & 291 & 89.26 & & \\
\hline Yes & 87 & 28.52 & 35 & 10.74 & 31.97 & $<0.001$ \\
\hline \multicolumn{7}{|c|}{ Tobacco consumption } \\
\hline Current or ever & 118 & 38.69 & 79 & 24.23 & & \\
\hline Never & 187 & 61.31 & 247 & 75.77 & 15.33 & $<0.001$ \\
\hline \multicolumn{7}{|c|}{ Alcohol consumption } \\
\hline Current or ever & 147 & 48.20 & 106 & 32.52 & & \\
\hline Never & 158 & 51.80 & 220 & 67.48 & 16.13 & $<0.001$ \\
\hline Cholesterol & $5.25 \pm 1.52$ & & $5.30 \pm 1.36$ & & 0.44 & 0.33 \\
\hline LDL & $3.56 \pm 1.24$ & & $3.05 \pm 1.15$ & & 5.36 & $<0.001$ \\
\hline $\mathrm{HDL}$ & $1.25 \pm 0.85$ & & $1.37 \pm 0.67$ & & 1.98 & 0.02 \\
\hline Triglycerides & $1.92 \pm 1.27$ & & $1.60 \pm 0.89$ & & 3.68 & $<0.001$ \\
\hline
\end{tabular}

$\mathrm{Cl}$ = cerebral infarction; $\mathrm{LDL}=$ low-density lipoprotein; $\mathrm{HDL}=$ high-density lipoprotein.

Table 2. Association between IL6 -174G/C ( $r$ 1800795) and -572C/G (rs1800796) polymorphisms and risk of cerebral infarction.

\begin{tabular}{|c|c|c|c|c|c|c|}
\hline Genotype & Patients $\mathrm{N}=305$ & $\%$ & Controls $\mathrm{N}=326$ & $\%$ & OR $(95 \% \mathrm{Cl})^{1}$ & $P$ value \\
\hline \multicolumn{7}{|l|}{ rs1800795 } \\
\hline GG & 196 & 64.26 & 243 & 74.54 & 1.0 (Ref) & - \\
\hline $\mathrm{GC}$ & 74 & 24.26 & 69 & 21.17 & $1.33(0.89-1.98)$ & 0.14 \\
\hline $\mathrm{CC}$ & 35 & 11.48 & 14 & 4.29 & $3.10(1.57-6.41)$ & $<0.001$ \\
\hline $\mathrm{GC}+\mathrm{CC}$ & 109 & 35.74 & 83 & 25.46 & $1.63(1.14-2.33)$ & 0.005 \\
\hline \multicolumn{7}{|l|}{ rs1800796 } \\
\hline GG & 117 & 38.36 & 139 & 42.64 & 1.0 (Ref) & - \\
\hline GC & 149 & 48.85 & 151 & 46.32 & $1.17(0.83-1.66)$ & 0.35 \\
\hline $\mathrm{CC}$ & 39 & 12.79 & 36 & 11.04 & $1.29(0.74-2.23)$ & 0.34 \\
\hline $\mathrm{GC}+\mathrm{CC}$ & 188 & 61.64 & 187 & 57.36 & $1.19(0.86-1.66)$ & 0.27 \\
\hline
\end{tabular}

${ }^{1}$ Adjusted for age, gender, body mass index, hypertension, diabetes mellitus, tobacco consumption, alcohol consumption, low-density lipoproteins, high-density lipoproteins, and triglycerides. OR = odds ratio; $95 \% \mathrm{Cl}=95 \%$ confidence interval; Ref $=$ reference .

\section{DISCUSSION}

It is well known that IL6 is an important mediator of in vivo inflammatory reactions and the inflammatory response to cerebral infarction, and is associated with atherosclerotic disease (Pola et al., 2003). In addition, a previous study reported that serum levels of this cytokine significantly increase in cerebral infarction (Acalovschi et al., 2003). Experimental studies have reported that SNPs in the non-coding promoter sequence of the IL6 gene can significantly affect its expression, and our study has indicated that the $-174 \mathrm{G} / \mathrm{C}$ (rs1800795) polymorphism, also present in its promoter, might influence the development of cerebral infarction.

Previous investigations have demonstrated a significant association between the $-174 \mathrm{G} / \mathrm{C}$ 
(rs1800795) polymorphism and risk of ischemic stroke, but their results have been inconsistent (Lalouschek et al., 2006; Strand et al., 2007; Yang et al., 2014; Bazina et al., 2015). Bazina et al. (2015) conducted a study with 144 ischemic stroke patients and 187 healthy controls, and identified a significant link between this polymorphism and an increased risk of ischemic stroke. Moreover, Yang et al. (2014) reported a case-control study in a Chinese population, from which they concluded that both $-174 \mathrm{G} / \mathrm{C}$ (rs1800795) and -572C/G (rs1800796) SNPs were associated with an elevated risk of ischemic stroke in young patients, and that these polymorphisms interact with other factors including hypertension, obesity, and etiologic subtype. In the present work, we found that the IL6 -174G/C (rs1800795) CC genotype and C allele are associated with an increased risk of cerebral infarction, a result that corresponds with the above-mentioned findings. However, Lalouschek et al. (2006) previously failed to establish a significant relationship between this polymorphism and the risk of ischemic cerebrovascular events. Likewise, Strand et al. (2007) reported no significant association between the -174G/C (rs1800795) polymorphism and first-ever stroke incidence. Such discrepancy between the results of previous studies may have been caused by differences in populations, study designs, and sample sizes.

There are several limitations to our study. First, all patients and control subjects were selected from one hospital, therefore selection bias may not have been avoided in this study. Second, in addition to IL6 -174G/C (rs1800795) and -572C/G (rs1800796), other polymorphisms may influence the development of cerebral infarction. Third, the small numbers of cases and controls enrolled may have limited the statistical power of this investigation. Therefore, further studies incorporating greater numbers of subjects are needed to clarify the association between IL6 -174G/C (rs1800795) and -572C/G (rs1800796) SNPs and the risk of cerebral infarction.

In conclusion, our study suggests that the CC genotype and $C$ allele of the $-174 \mathrm{G} / \mathrm{C}$ (rs1800795) polymorphism are associated with an increased risk of cerebral infarction. Further welldesigned research efforts involving large sample sizes are greatly needed to confirm our results.

\section{Conflicts of interest}

The authors declare no conflict of interest.

\section{REFERENCES}

Acalovschi D, Wiest T, Hartmann M, Farahmi M, et al. (2003). Multiple levels of regulation of the interleukin-6 system in stroke. Stroke 34: 1864-1869.

Andreotti F, Porto I, Crea F and Maseri A (2002). Inflammatory gene polymorphisms and ischemic heart disease: review of population association studies. Heart 87: 107-112.

Balding J, Livingstone WJ, Pittock SJ, Mynett-Johnson L, et al. (2004). The IL-6 G-174C polymorphism may be associated with ischaemic stroke in patients without a history of hypertension. Ir. J. Med. Sci. 173: 200-203.

Bazina A, Sertić J, Mišmaš A, Lovrić T, et al. (2015). PPARy and IL-6 -174G>C gene variants in Croatian patients with ischemic stroke. Gene 560: 200-204.

Dichgans M (2007). Genetics of ischemic stroke. Lancet Neurol. 6: 149-161.

Donnan GA, Fisher M, Macleod M and Davis SM (2008). Stroke. Lancet 371: 1612-1623.

Ionita CC, Xavier AR, Kirmani JF, Dash S, et al. (2005). What proportion of stroke is not explained by classic risk factors? Prev. Cardiol. 8: 41-46.

Lalouschek W, Schillinger M, Hsieh K, Endler G, et al. (2006). Polymorphisms of the inflammatory system and risk of ischemic cerebrovascular events. Clin. Chem. Lab. Med. 44: 918-923.

Liu M, Wu B, Wang WZ, Lee LM, et al. (2007). Stroke in China: epidemiology, prevention, and management strategies. Lancet Neurol. 6: 456-464. 
Liu L, Wang D, Wong KS and Wang Y (2011). Stroke and stroke care in China: huge burden, significant workload, and a national priority. Stroke 42: 3651-3654.

Meschia JF, Worrall BB and Rich SS (2011). Genetic susceptibility to ischemic stroke. Nat. Rev. Neurol. 7: $369-378$.

Pola R, Flex A, Gaetani E, Flore R, et al. (2003). Synergistic effect of -174 G/C polymorphism of the interleukin- 6 gene promoter and $469 \mathrm{E} / \mathrm{K}$ polymorphism of the intercellular adhesion molecule-1 gene in Italian patients with history of ischemic stroke. Stroke 34: 881-885.

Qi XF, Feng TJ, Yang P, Feng HY, et al. (2014). Role of inflammatory parameters in the susceptibility of cerebral thrombosis. Genet. Mol. Res. 13: 6350-6355.

Saiki R, Hayashi D, Ikuo Y, Nishimura K, et al. (2013). Acrolein stimulates the synthesis of IL-6 and C-reactive protein (CRP) in thrombosis model mice and cultured cells. J. Neurochem. 127: 652-659.

Schoester M, Heinrich PC and Graeve L (1994). Regulation of interleukin-6 receptor expression by interleukin-6 in human monocytes-a re-examination. FEBS Lett. 345: 131-134.

Shenhar-Tsarfaty S, Ben Assayag E, Bova I, Shopin L, et al. (2010). Interleukin-6 as an early predictor for one-year survival following an ischaemic stroke/transient ischaemic attack. Int. J. Stroke 5: 16-20.

Stoll G and Bendszus M (2006). Inflammation and atherosclerosis: novel insights into plaque formation and destabilization. Stroke 37: 1923-1932.

Strand M, Soderstrom I, Wiklund PG, Hallmans G, et al. (2007). Polymorphisms at the osteoprotegerin and interleukin-6 genes in relation to first-ever stroke. Cerebrovasc. Dis. 24: 418-425.

Yamada Y, Metoki N, Yoshida H, Satoh K, et al. (2006). Genetic risk for ischemic and hemorrhagic stroke. Arterioscler. Thromb. Vasc. Biol. 26: 1920-1925.

Yang X, Feng L, Li C and Li Y (2014). Association of IL-6-174G > C and -572C > G polymorphisms with risk of young ischemic stroke patients. Gene 539: 258-262. 